\title{
A compact fourth-order spatial discretisation applied to the Navier-Stokes equations L. Zhou ${ }^{1} \quad$ S. W. Armfield ${ }^{2}$
}

(Received 10 March 2015; revised 16 May 2016)

\begin{abstract}
Modern direct and large eddy simulation of turbulent and transition flows requires accurate solution of the Navier-Stokes equations. High accuracy is achieved using a high order discretisation. The standard high order approach for local methods, such as finite-difference or finitevolume, produces large computational molecules and thus introduces complexity in the boundary treatment and parallelisation. Existing compact schemes need to invert a matrix system, which increases the computational cost, and are restricted to application on non-uniform grids. The fourth-order compact scheme proposed here iteratively applies a low order compact method to achieve higher accuracy. The scheme allows for a simple application of boundary conditions, can be applied on a non-uniform grid and allows a standard parallelisation
\end{abstract}

http://journal.austms.org.au/ojs/index.php/ANZIAMJ/article/view/9422 gives this article, (C) Austral. Mathematical Soc. 2016. Published May 30, 2016, as part of the Proceedings of the 17th Biennial Computational Techniques and Applications Conference. ISSN 1446-8735. (Print two pages per sheet of paper.) Copies of this article must not be made otherwise available on the internet; instead link directly to this URL for this article. 
approach to be used. The scheme is implemented and tested in an unsteady finite-difference heat equation solver and benchmarked against the analytical solution to validate the order of accuracy. It is also included in a full fractional-step Navier-Stokes solver and validated for the lid-driven cavity problem.

\section{Contents}

1 Introduction

C482

2 Numerical methods $\quad \mathbf{C 4 8 4}$

2.1 Spatial scheme . . . . . . . . . . . . . . C484

2.2 Time scheme . . . . . . . . . . . . . . . . . . C C487

2.3 Grids . . . . . . . . . . . . . . . . . C487

3 Applications $\quad \mathbf{C 4 8 8}$

3.1 Solutions of the heat equation . . . . . . . . . . C C488

3.2 Solutions of the Navier-Stokes equations . . . . . . . . C491

4 Summary

C498

References

C498

\section{Introduction}

The governing equations for almost all computational fluid dynamics problems are the Navier-Stokes equations shown in equation (12) to (14). Starting from the Chorin scheme [1], a variety of numerical approaches have been developed to solve these equations. With the increasing demand for accurate solutions of the Navier-Stokes equations required in modern direct or largeeddy simulations for turbulent and transition flow, the development of high 
order spatial schemes is of great significance, improving solution accuracy, and requiring less computational power.

The simplest form of a high order scheme, the standard central difference scheme (CDS) requires large computational molecules [2], making it difficult to properly evaluate the derivatives at nodes near boundaries. Gibou and Fedkiw [3] used a fourth-order central difference scheme (CDS-4) and suggested a boundary treatment using ghost cells. By adding extra nodes external to the computational domain, derivatives at all interior nodes can be evaluated once nodal values are known. Unfortunately, the values at the external nodes are undefined and must be obtained by extrapolation. Maintaining fully fourthorder behaviour with the CDS-4 scheme requires sixth-order extrapolation for the external nodes, further affecting the complexity and efficiency of the code. The large computational molecules also reduce the efficiency of parallelisation, requiring a greater exchange of data on the subdomain boundaries.

Compact schemes reduce the size of the computational molecule so that the solution at any node depends directly only on the solution at neighbour nodes. Lele [4] proposed such a scheme where higher order approximations to the spatial derivatives were obtained by solving an additional matrix system for those derivatives at each node. This increased the computational cost while the solution accuracy was severely degraded for non-uniform grids [2]. Despite these disadvantages this approach was used by a number of researches [5, 6, 7].

The scheme proposed in this article provides fourth-order spatial accuracy in a compact finite difference form. The high order accuracy is achieved by iteratively applying low order discretisations. The lower order discretisation is chosen to be the second-order central difference scheme (CDS-2) which does not require the solution of an additional linear system. The new spatial scheme was coded and validated in a typical two-dimensional steady-state heat conduction problem. It was also applied in a full unsteady two-dimensional incompressible Navier-Stokes solver for the lid-driven cavity problem. 


\section{Numerical methods}

\section{$2.1 \quad$ Spatial scheme}

Approximation of first derivative Consider the case of a one-dimensional function $\phi(x)$ defined on a uniformly-discretised domain with grid size $h$, with $x_{i}$ the nodal locations. Using Taylor series, the first derivative $\phi_{x}$ is approximated in a second central difference form for the whole computational domain by

$$
\phi_{i}^{\prime}=\frac{1}{h}\left(-\frac{1}{2} \phi_{i-1}+\frac{1}{2} \phi_{i+1}\right)=\phi_{x}+\frac{h^{2}}{6} \phi^{(3)}+\frac{h^{4}}{120} \phi^{(5)}+\mathcal{O}\left(h^{6}\right),
$$

where $\phi_{i}^{\prime}$ is a second-order central difference approximation to the first derivative of $\phi$, at the $x_{i}$ location, and $\phi_{x}$ is the exact first derivative of $\phi$ at $x_{i}$. The second-order central derivative approximation at $x_{i}$ is $\phi_{i}^{\prime \prime}$ and the exact second derivative is $\phi_{x x}$. Higher derivatives are denoted as $\phi^{(\mathfrak{n})}$, representing the $n$th derivatives of $\phi$ at $x_{i}$.

A linear combination of the $\phi^{\prime}$ terms at $i-1, i$ and $i+1$ provides a fourth-order accurate approximation for the first derivative of $\phi$ at $x_{i}$ as

$$
\hat{\phi}_{i}^{\prime}=\alpha \phi_{i-1}^{\prime}+\beta \phi_{i}^{\prime}+\gamma \phi_{i+1}^{\prime}=\phi_{x}+\mathcal{O}\left(h^{4}\right),
$$

where the coefficients $\alpha, \beta$ and $\gamma$ are obtained by expanding the second-order finite difference form at $i-1, i$ and $i+1$, and setting the $h, h^{2}$ and $h^{3}$ coefficients to zero. Solving for $\alpha, \beta$ and $\gamma$ gives

$$
\hat{\phi}_{i}^{\prime}=-\frac{1}{6} \phi_{i-1}^{\prime}+\frac{4}{3} \phi_{i}^{\prime}-\frac{1}{6} \phi_{i+1}^{\prime} .
$$

In this process of obtaining a fourth-order discretisation, instead of a fivepoint stencil [8] performed once over the entire domain, a three-point stencil is applied twice. As a result, the computational cost for the fourth-order compact scheme (Compact-4) is expected to be twice that of CDS-2. 
Approximation of second derivative The second derivative is approximated similarly to the first derivative. The central difference form for the second derivative at location $i$ is

$$
\phi_{i}^{\prime \prime}=\frac{1}{h^{2}}\left(\phi_{i-1}-2 \phi_{i}+\phi_{i+1}\right)=\phi_{x x}+\frac{h^{2}}{12} \phi^{(4)}+\frac{h^{4}}{360} \phi^{(6)}+\mathcal{O}\left(h^{8}\right) .
$$

Assuming that the fourth-order discretisation is a linear combination of the above discretisation gives

$$
\hat{\phi}_{i}^{\prime \prime}=\alpha \phi_{i-1}^{\prime \prime}+\beta \phi_{i}^{\prime \prime}+\gamma \phi_{i+1}^{\prime \prime}=\phi_{x x}+\mathcal{O}\left(h^{4}\right) .
$$

Requiring all terms with order lower than $\mathrm{h}^{4}$ to be zero gives

$$
\hat{\phi}_{i}^{\prime \prime}=-\frac{1}{12} \phi_{i-1}^{\prime \prime}+\frac{7}{6} \phi_{i}^{\prime \prime}-\frac{1}{12} \phi_{i+1}^{\prime \prime} .
$$

Boundary treatment and truncation error To obtain the fourth-order approximations to the derivatives at all interior nodes, boundary derivatives are used rather than boundary values. Boundary derivatives require a fourthorder extrapolation for the derivatives at the boundary nodes. The standard Lagrange interpolating polynomial approach given in by Kudryavtsev and Hazewinkel [9] is used here. The higher the order of extrapolation, the more nodes required. The extrapolation is applied after obtaining $\phi^{\prime}$ and $\phi^{\prime \prime}$ at the interior nodes to provide those quantities on the boundaries. Provided at least fourth-order extrapolation is used, $\hat{\phi}^{\prime}$ and $\hat{\phi}^{\prime \prime}$ will retain their fourth-order accuracy. Using higher order extrapolations will alter the magnitude of the coefficients of the truncation terms, but does not change the overall order of accuracy, as shown in Table 1.

Table 1 compares the truncation error of the compact fourth-order scheme, described above and denoted Compact-4, to that of the standard second and fourth-order central schemes, CDS-2 and CDS-4, and to the compact Padé approximation scheme of Lele [4], Padé-4. Peter and Chenwu [7] provided the truncation error terms for Padé-4. 
Table 1: Truncation terms for approximations at boundary nodes using Compact-4.

\begin{tabular}{l|lr|lr} 
Extrapolations & \multicolumn{2}{|c|}{ First Derivative } & \multicolumn{2}{c}{ Second Derivative } \\
\hline 4 th order & $-\frac{\phi^{(5)}}{30} h^{4}-\frac{\phi^{(6)}}{6} h^{5}+\mathcal{O}\left(h^{6}\right)$ & $-\frac{\phi^{(6)}}{90} h^{4}-\frac{\phi^{(7)}}{12} h^{5}+\mathcal{O}\left(h^{6}\right)$ \\
5 th order & $-\frac{\phi^{(5)}}{30} h^{4}$ & $+\mathcal{O}\left(h^{6}\right)$ & $-\frac{\phi^{(6)}}{90} h^{4}$ & $+\mathcal{O}\left(h^{6}\right)$ \\
6th order & $-\frac{\phi^{(5)}}{30} h^{4}$ & $+\mathcal{O}\left(h^{6}\right)$ & $-\frac{\phi^{(6)}}{90} h^{4}$ & $+\mathcal{O}\left(h^{6}\right)$
\end{tabular}

Table 2: Truncation error for $\phi_{x}$ and $\phi_{x x}$.

\begin{tabular}{l|rc|rc} 
& \multicolumn{2}{|c|}{ First } & Derivative & \multicolumn{2}{c}{ Second Derivative } \\
Scheme & Interior & Boundary & Interior & Boundary \\
\hline CDS-2 & $\frac{\phi^{(3)}}{6} h^{2}$ & - & $\frac{\phi^{(4)}}{12} h^{2}$ & - \\
CDS-4 & $-\frac{\phi^{(5)}}{30} h^{4}$ & $-\frac{\phi^{(5)}}{30} h^{4}-\frac{\phi^{(6)}}{12} h^{5}$ & $-\frac{\phi^{(6)}}{90} h^{4}$ & $-\frac{\phi^{(6)}}{90} h^{4}-\frac{\phi^{(7)}}{12} h^{5}$ \\
Padé-4 & $-\frac{\phi^{(5)}}{120} h^{4}$ & - & $\frac{\phi^{(6)}}{20} h^{4}$ & - \\
Compact-4 & $-\frac{\phi^{(5)}}{30} h^{4}$ & $-\frac{\phi^{(5)}}{30} h^{4}-\frac{\phi^{(6)}}{6} h^{5}$ & $-\frac{\phi^{(6)}}{90} h^{4}$ & $-\frac{\phi^{(6)}}{90} h^{4}-\frac{\phi^{(7)}}{12} h^{5}$
\end{tabular}

The Compact-4 scheme provides the same order of accuracy as CDS-4 but is more compact in form since it uses a three-node molecule. Compact-4 requires only fourth-order extrapolation for the derivatives at the boundary but provides similar accuracy to CDS- 4 at the boundaries. In contrast, the CDS-4 scheme requires fifth-order extrapolation for the first derivative and sixth-order for the second derivative. Both the high order of the polynomial and the varied implementation complicate the application and parallelisation of CDS-4. Additionally, the Compact- 4 scheme does not require the inversion of a matrix system required in Padé- 4 . 


\subsection{Time scheme}

To obtain the fully developed steady state solution, an explicit Euler time advancing method is applied as

$$
\frac{\phi^{n+1}-\phi^{n}}{\Delta t}=\operatorname{RHS}^{n},
$$

where superscript $n$ denotes the time step and RHS is the right hand side of either the heat equation (8) or the momentum equations (12) and (13). The solution is then advanced in time and is fully developed in Section 3.

\subsection{Grids}

Both uniform and non-uniform grids are considered. In some problems where the solution varies significantly near boundaries, for example boundary layer flows, non-uniform grids are more effective. Since the grid size is not a flow constant, schemes on non-uniform grids have lower order accuracy than on uniform grids. The formal orders of accuracy of the schemes described here are reduced on non-uniform grids [10, 11, 12], as are the convergence rates. Also, the solution accuracy and convergence is dependent on the aspect ratio of the grid [13].

The non-uniform grids chosen here are symmetrically expanded with a constant expansion ratio, as shown in Figure 1. Such grids can be refined by inserting extra nodes between any two nodes at a place where the refined grid also has a constant ratio of spacings. As a result, the expansion factor of the fine grid is the square root of that of the coarse grid, and the order of accuracy is expected to be, asymptotically, the same as that of the uniform grid [2]. The initial grid has 20 nodes in both directions with an expansion ratio of 1.1, which is the case shown in the Figure 1. 
Figure 1: Non-uniform grid example $(\mathrm{N}=20, \mathrm{r}=1.1)$.

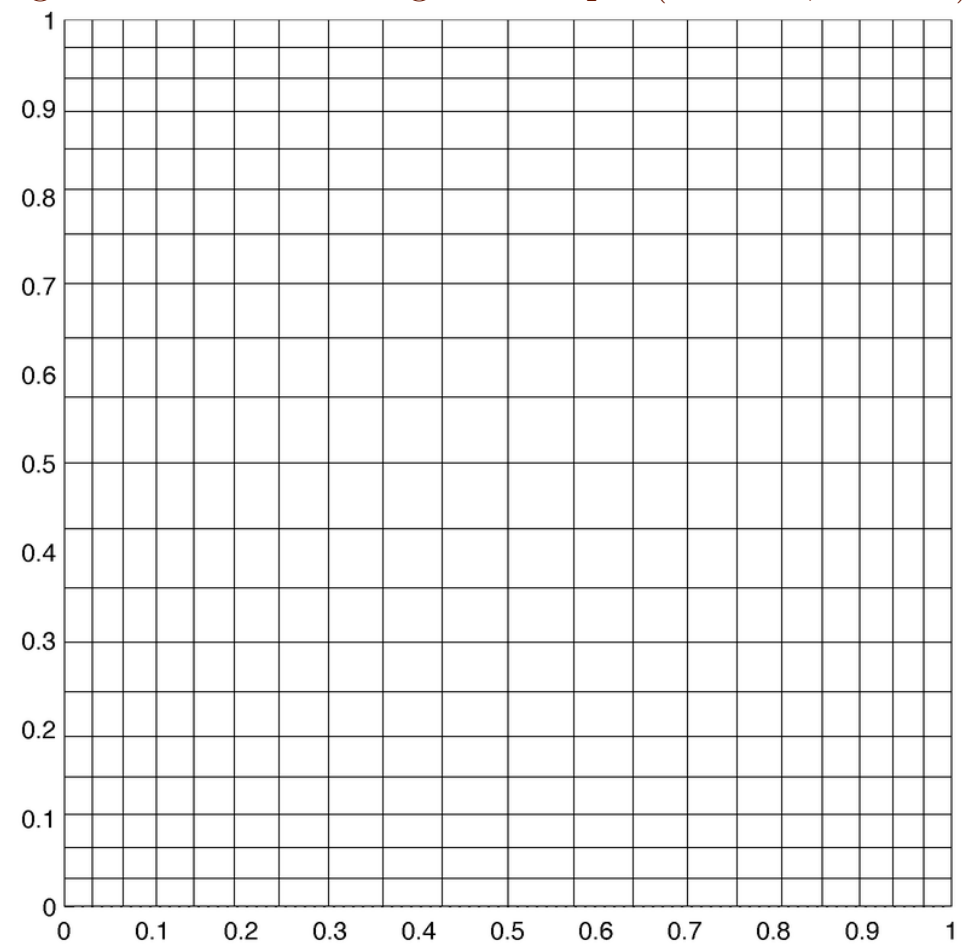

\section{Applications}

The schemes were applied to the two-dimensional heat conduction problem and the lid-driven cavity problem with Reynolds number of 100, where the order of accuracy was validated on both uniform and non-uniform grids.

\subsection{Solutions of the heat equation}

The heat equation is a linear parabolic partial differential equation. The specific heat equation that is tested here was chosen due to the availability of 
the exact solution that is used to validate the accuracy of the schemes,

$$
T_{t}=k\left(T_{x x}+T_{y y}\right)+\sin (\pi y) \sin (3 \pi x) \quad \text { on } \Omega=[0,1] \times[0,1],
$$

where the diffusivity $\mathrm{K}=0.01, \mathrm{~T}$ is the temperature field, and all angles are in radians. Zero Dirichlet boundary conditions are specified on all sides of the domain, and the initial condition is zero. The fully developed exact solution is

$$
\mathrm{T}=\frac{10}{\pi^{2}} \sin (\pi y) \sin (3 \pi x) .
$$

To ensure the time advancing scheme is stable, the time step is constrained using the von Neumann stability condition [14, 15]

$$
\Delta t \leqslant \frac{h^{2}}{4 \kappa}
$$

for CDS-2, and

$$
\Delta t \leqslant \frac{h^{2}}{8 k},
$$

for CDS-4 and Compact-4. The solution is considered to have reached its steady state when the residual, obtained by averaging the unsteady term $\mid\left(T^{n+1}-\right.$ $\left.\mathrm{T}^{\mathrm{n}}\right) / \Delta \mathrm{t} \mid$ over the domain, reduces to $1 \times 10^{-8}$.

Grid tests were carried out by examining the error, shown in Table 3 at a specific node when the grid was refined. The node chosen here is the centre of the domain with coordinates $(0.5,0.5)$. The analytical solution is $\mathrm{T}(0.5,0.5)=-10 / \pi^{2}$. The CDS- 4 results are not listed here because they are identical to the Compact-4 results as the two schemes have identical truncation error for second derivatives.

Table 3 shows that Compact- 4 is capable of providing an accurate solution on a much coarser grid compared to the second order scheme. The solution $\mathrm{T}(0.5,0.5)$ on a $40 \times 40$ grid using Compact- 4 is even more accurate than that on a $320 \times 320$ grid using CDS- 2 . The order of accuracy calculation 
Table 3: Error tests for steady-state $\mathrm{T}(0.5,0.5)$.

\begin{tabular}{rrrrr} 
& \multicolumn{2}{c}{ uniform grids } & \multicolumn{2}{c}{ non-uniform grids } \\
$\mathrm{N}$ & CDS-2 & Compact-4 & CDS-2 & Compact-4 \\
\hline 20 & $-1.7 \times 10^{-2}$ & $-8.7 \times 10^{-4}$ & $-9.2 \times 10^{-3}$ & $3.1 \times 10^{-3}$ \\
40 & $-4.3 \times 10^{-3}$ & $-3.4 \times 10^{-5}$ & $-1.9 \times 10^{-3}$ & $2.3 \times 10^{-4}$ \\
80 & $-1.1 \times 10^{-3}$ & $-2.0 \times 10^{-6}$ & $-4.6 \times 10^{-4}$ & $1.5 \times 10^{-5}$ \\
160 & $-2.6 \times 10^{-4}$ & $-9.8 \times 10^{-8}$ & $-1.1 \times 10^{-4}$ & $9.3 \times 10^{-7}$ \\
320 & $-6.4 \times 10^{-5}$ & $-1.7 \times 10^{-8}$ & $-2.9 \times 10^{-5}$ & $8.6 \times 10^{-8}$ \\
\hline $\mathcal{O}\left(\mathrm{h}^{\mathfrak{n}}\right)$ & 2.0005 & 4.0206 & 2.0158 & 4.0587
\end{tabular}

confirms that the Compact- 4 scheme is fourth-order accurate in space. Figure 2 shows the error relative to the exact solution at different grid numbers. Errors of solutions using Compact-4 decrease much faster as the grid number increases than those obtained with CDS-2. The decrease of error follows the fourth-order fitting line, also demonstrating the Compact- 4 scheme is fourth-order accurate in space.

In terms of $\mathrm{CPU}$ time performance, comparison was made between two cases: Compact- 4 on the $80 \times 80$ non-uniform grid and CDS-2 on the $160 \times 160$ non-uniform grid. Figure 2 shows that these two cases have similar accuracy, but the CPU time for the former is around 210 seconds and for the latter is 1100 seconds, which is about four times longer. CPU time tests were run in Matlab R2014b on a single core of an i7 $9603.2 \mathrm{GHz}$ machine. 
Figure 2: Heat equation solution convergence.

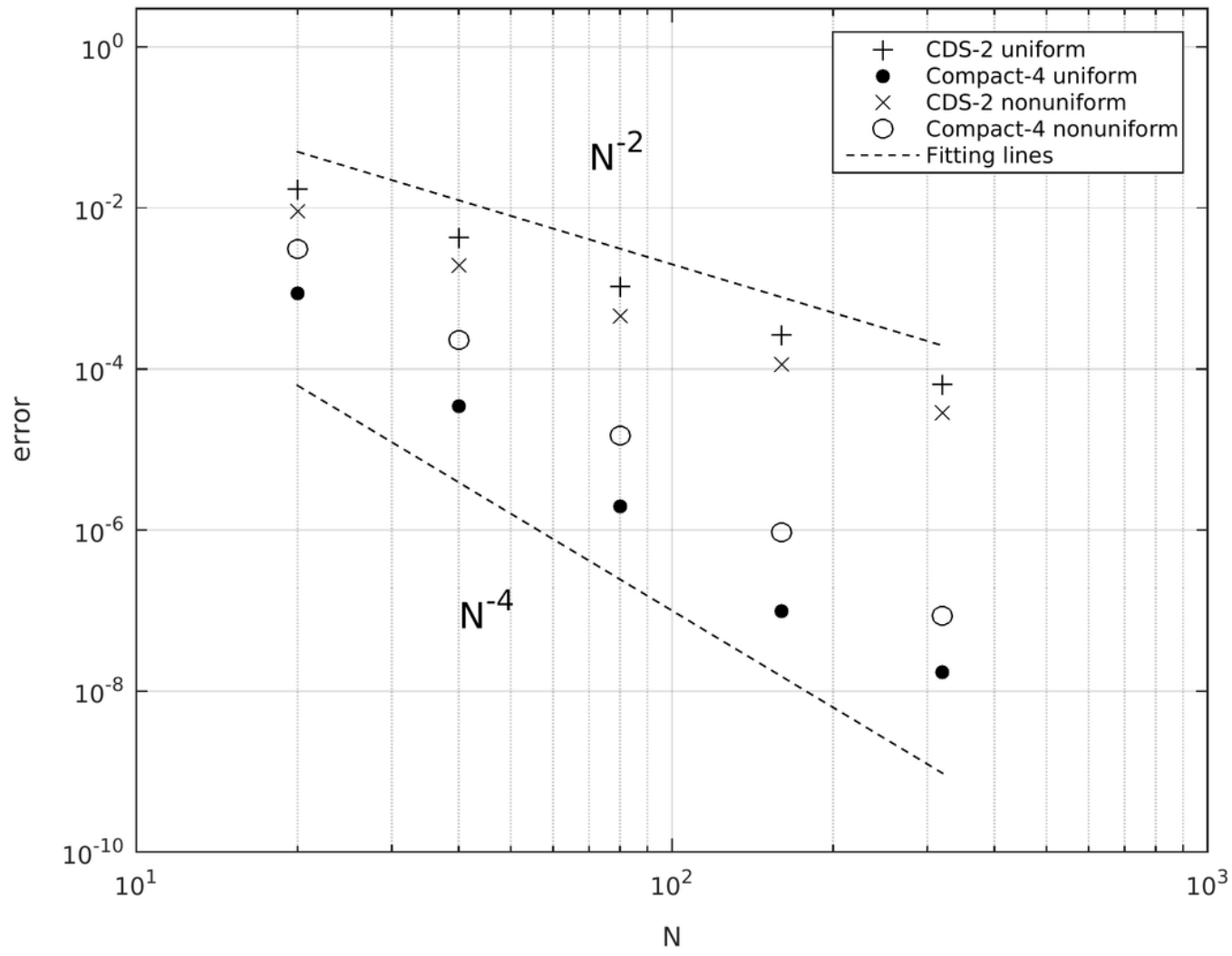

\subsection{Solutions of the Navier-Stokes equations}

The governing equations for the lid-driven cavity flow are the two-dimensional incompressible unsteady Navier-Stokes equations,

$$
\begin{aligned}
u_{t}+u_{x}+u_{y} & =-P_{x}+\frac{1}{R e}\left(u_{x x}+u_{y y}\right) \\
v_{t}+u v_{x}+V_{y} & =-P_{y}+\frac{1}{R e}\left(v_{x x}+V_{y y}\right), \\
u_{x}+V_{y} & =0
\end{aligned}
$$


where $\mathrm{U}$ and $\mathrm{V}$ are velocities in the $\mathrm{x}$ and $\mathrm{y}$ directions, respectively, $\mathrm{P}$ is the pressure, $R e=\bar{V} L / v$ is the Reynolds number, where $\mathrm{V}$ is the mean driving velocity, $L$ is the cavity width, and $v$ is the kinematic viscosity of the fluid. The lid-driven cavity problem is a common benchmark flow for scheme development due to its simple geometry and boundary conditions. The equations are solved in domain $\Omega=[0,1] \times[0,1]$. The side $y=1$ for all $x$ has a shearing velocity that drives the cavity flow, with the velocity on all other boundaries set to zero. The Reynolds number is set to 100. Benchmark results are widely available for low Reynolds number flow [16, 17, 18, 19]. To avoid velocity singularities at the two corners $(x, y)=(0,1)$ and $(1,1)$, the regularised driven cavity is applied when the driving velocity is smoothed by applying a continuous distribution [20]. The shearing velocity in this case takes the distribution of a parabola, with an average magnitude of 1.0,

$$
u_{y=1}=-6 x^{2}+6 x,
$$

where $x=0$ at the left hand side of the domain.

The fractional step method has been widely used and examined for the solution of the unsteady incompressible Navier-Stokes equations on both staggered and non-staggered grids [21, 22, 23]. The fractional step method used here is explicit and is applied on a non-staggered grid. It is briefly written as

$$
\begin{aligned}
& \frac{\mathrm{U}^{*}-\mathrm{U}^{\mathrm{n}}}{\Delta \mathrm{t}}+\left(\mathrm{uU}_{\mathrm{x}}+\mathrm{Vu}_{\mathrm{y}}\right)^{\mathrm{n}}=-\mathrm{P}_{\mathrm{x}}^{\mathrm{n}}+\frac{1}{\operatorname{Re}}\left(\mathrm{u}_{\mathrm{xx}}+\mathrm{U}_{y y}\right)^{\mathrm{n}} \text {, } \\
& \frac{\mathrm{V}^{*}-\mathrm{V}^{\mathrm{n}}}{\Delta \mathrm{t}}+\left(\mathrm{U} \mathrm{V}_{\mathrm{x}}+\mathrm{WV}_{\mathrm{y}}\right)^{\mathrm{n}}=-\mathrm{P}_{\mathrm{y}}^{\mathrm{n}}+\frac{1}{\operatorname{Re}}\left(\mathrm{V}_{\mathrm{xx}}+\mathrm{V}_{y y}\right)^{\mathrm{n}} \text {, } \\
& \Pi_{x x}+\Pi_{y y}=\frac{1}{\Delta t}\left(U_{x}^{*}+V_{y}^{*}\right) \text {, } \\
& \mathrm{P}^{\mathrm{n}+1}=\mathrm{P}^{\mathrm{n}}+\Pi \text {, } \\
& \mathrm{U}^{\mathrm{n}+1}=\mathrm{U}^{*}-\Delta \mathrm{t} \Pi_{\mathrm{x}} \text {, } \\
& \mathrm{V}^{\mathrm{n}+1}=\mathrm{V}^{*}-\Delta \mathrm{t} \Pi_{\mathrm{y}},
\end{aligned}
$$

where $\mathrm{U}^{*}$ and $\mathrm{V}^{*}$ are explicit solutions of the momentum equations (12) and (13), and $\Pi$ is the pressure correction term. 
The application of Compact-4 to all spatial derivatives in the fractional-step scheme is straight forward, with the exception of the $\Pi_{x x}+\Pi_{y y}$ terms in the pressure correction equation (18). As this is an implicit equation which is solved for $\Pi$, the discretisation must be in terms of $\Pi$, and therefore we can use either CDS-2 or CDS-4. To maintain the overall compact structure of the algorithm, it is better to use the CDS-2 scheme. The effect of the CDS-2 scheme on the overall accuracy of the scheme is examined below. In a one-dimensional analogue of (18), applying the Compact- 4 scheme to the divergence terms and a second-order central difference scheme to the pressure correction term, we obtain

$$
\Pi_{i}^{\prime \prime}=\frac{\Pi_{i-1}-2 \Pi_{i}+\Pi_{i+1}}{h^{2}}=\frac{1}{\Delta t} \hat{u}_{i}^{\prime *}=\frac{1}{\Delta t}\left(-\frac{1}{6}{u_{i-1}^{\prime *}}^{*}+\frac{4}{3} u_{i}^{\prime *}-\frac{1}{6} u_{i+1}^{\prime *}\right),
$$

and the correction to the velocities is discretised as

$$
\mathrm{U}_{\mathrm{i}}^{\mathrm{n}+1}=\mathrm{U}_{\mathrm{i}}^{*}-\Delta \mathrm{t}\left(-\frac{1}{6} \Pi^{\prime}{ }_{i-1}+\frac{4}{3} \Pi_{i}^{\prime}-\frac{1}{6} \Pi_{i+1}^{\prime}\right) .
$$

Taking the first derivative of equation (23), applying the Compact-4 scheme to the $\hat{\Pi}^{\prime}$ terms, and multiplying equation (22) by $\Delta t$ to remove the $\hat{\mathrm{U}}_{i}^{*}{ }^{*}$ from equation (23), gives

$$
\begin{aligned}
\mathrm{U}_{i}^{\mathrm{n}+1}= & \Delta \mathrm{t} \Pi^{\prime \prime}{ }_{i}-\Delta \mathrm{t}\left[-\frac{1}{6}\left(-\frac{1}{6} \Pi^{\prime \prime}{ }_{i-2}+\frac{4}{3} \Pi_{i-1}^{\prime \prime}-\frac{1}{6} \Pi^{\prime \prime}{ }_{i}\right)\right. \\
& +\frac{4}{3}\left(-\frac{1}{6} \Pi^{\prime \prime}{ }_{i-1}+\frac{4}{3} \Pi^{\prime \prime}{ }_{i}-\frac{1}{6} \Pi^{\prime \prime}{ }_{i+1}\right) \\
& \left.-\frac{1}{6}\left(-\frac{1}{6} \Pi^{\prime \prime}{ }_{i}+\frac{4}{3} \Pi^{\prime \prime}{ }_{i+1}-\frac{1}{6} \Pi^{\prime \prime}{ }_{i+2}\right)\right] \\
= & \Delta t \Pi^{\prime \prime}{ }_{i}-\Delta \mathrm{t}\left(\frac{1}{36} \Pi^{\prime \prime}{ }_{i-2}-\frac{4}{9} \Pi^{\prime \prime}{ }_{i-1}+\frac{11}{6} \Pi^{\prime \prime}{ }_{i}-\frac{4}{9} \Pi^{\prime \prime}{ }_{i+1}-\frac{1}{36} \Pi^{\prime \prime}{ }_{i+2}\right) \\
= & \Delta t\left(-\frac{1}{36} \Pi^{\prime \prime}{ }_{i-2}+\frac{4}{9} \Pi^{\prime \prime}{ }_{i-1}-\frac{5}{6} \Pi^{\prime \prime}{ }_{i}+\frac{4}{9} \Pi^{\prime \prime}{ }_{i+1}-\frac{1}{36} \Pi^{\prime \prime}{ }_{i+2}\right) .
\end{aligned}
$$


All $\Pi^{\prime \prime}$ terms are then expanded using CDS-2,

$$
\begin{aligned}
\mathrm{u}_{\mathrm{i}}^{\mathrm{n}+1} & =\frac{\Delta \mathrm{t}}{\mathrm{h}^{2}}\left(-\frac{1}{36} \Pi_{i-3}+\frac{1}{2} \Pi_{i-2}-\frac{7}{4} \Pi_{i-1}+\frac{23}{9} \Pi_{i}-\frac{7}{4} \Pi_{i+1}+\frac{1}{2} \Pi_{i+2}-\frac{1}{36} \Pi_{i+3}\right) \\
& =\frac{\Delta \mathrm{t}}{\mathrm{h}^{2}}\left(\frac{\mathrm{h}^{4}}{3} \Pi^{(4)}+\frac{\mathrm{h}^{6}}{36} \Pi^{(6)}+\mathcal{O}\left(\mathrm{h}^{8}\right)\right)=\frac{\Delta \mathrm{t}}{3} \mathrm{~h}^{2}\left[\Pi^{(4)}+\mathcal{O}\left(\mathrm{h}^{2}\right)\right] .
\end{aligned}
$$

Applying the same method to (23), but using a fourth-order central difference scheme for the pressure correction term, gives

$$
\begin{aligned}
\mathrm{u}_{\mathrm{i}}^{\mathrm{n}+1}= & \frac{\Delta \mathrm{t}}{\mathrm{h}^{2}}\left(-\frac{1}{36} \Pi_{i-3}+\frac{5}{12} \Pi_{i-2}-\frac{17}{12} \Pi_{i-1}+\frac{37}{18} \Pi_{i}-\frac{17}{12} \Pi_{i+1}\right. \\
& \left.+\frac{5}{12} \Pi_{i+2}-\frac{1}{36} \Pi_{i+3}\right) \\
= & \frac{\Delta \mathrm{t}}{\mathrm{h}^{2}}\left(\frac{\mathrm{h}^{4}}{4} \Pi^{(4)}+\frac{\mathrm{h}^{6}}{72} \Pi^{(6)}+\mathcal{O}\left(\mathrm{h}^{8}\right)\right)=\frac{\Delta \mathrm{t}}{4} \mathrm{~h}^{2}\left[\Pi^{(4)}+\mathcal{O}\left(\mathrm{h}^{2}\right)\right] .
\end{aligned}
$$

In equation (25) the velocity $\mathrm{U}$, after correction using CDS-2 for the pressure correction term, is fourth-order in space and second-order in time. Instead applying CDS-4 to the pressure correction term produces the same order but a smaller coefficient of $\Pi^{(4)}$, which implies that the discretisation of the pressure correction term $\Pi$ does not affect the order of accuracy of the corrected velocity term. Spatial accuracy tests were conducted on both uniform and non-uniform grids with pressure correction terms expanded by CDS-2 and CDS- 4 . Table 4 shows $\mathbf{U}(0.5,0.5)$ at different grid numbers. The residuals for the steady-state solutions, obtained by averaging the absolute unsteady terms in both the momentum equations (12) and (13), are both less than $1 \times 10^{-8}$. In all cases, zero normal derivative boundary conditions on all boundaries are applied for $\Pi$.

From Table 4, CDS-2 for the pressure correction terms gives steady-state solutions very close to CDS-4, confirming that the discretisation for pressure correction does not affect the solution accuracy when the steady-state solution 
Table 4: Steady-state $\mathrm{U}(0.5,0.5)$ pressure correction discretisation. uniform grids non-uniform grids

\begin{tabular}{rrrrr}
$\mathrm{N}$ & CDS-2 & CDS-4 & CDS-2 & CDS-4 \\
\hline 20 & -0.2561 & -0.2561 & -0.2633 & -0.2633 \\
40 & -0.2670 & -0.2670 & -0.2671 & -0.2671 \\
80 & -0.2674 & -0.2674 & -0.2674 & -0.2674 \\
160 & -0.2675 & -0.2675 & -0.2675 & -0.2675 \\
\hline $\mathcal{O}\left(\mathrm{h}^{\mathfrak{n}}\right)$ & 3.6247 & 3.6247 & 3.0448 & 3.0448
\end{tabular}

Figure 3: Quadratically distributed velocity driven cavity streamlines $(\mathrm{Re}=$ $100, \mathrm{~N}=160, \mathrm{r}=1.012$ ).

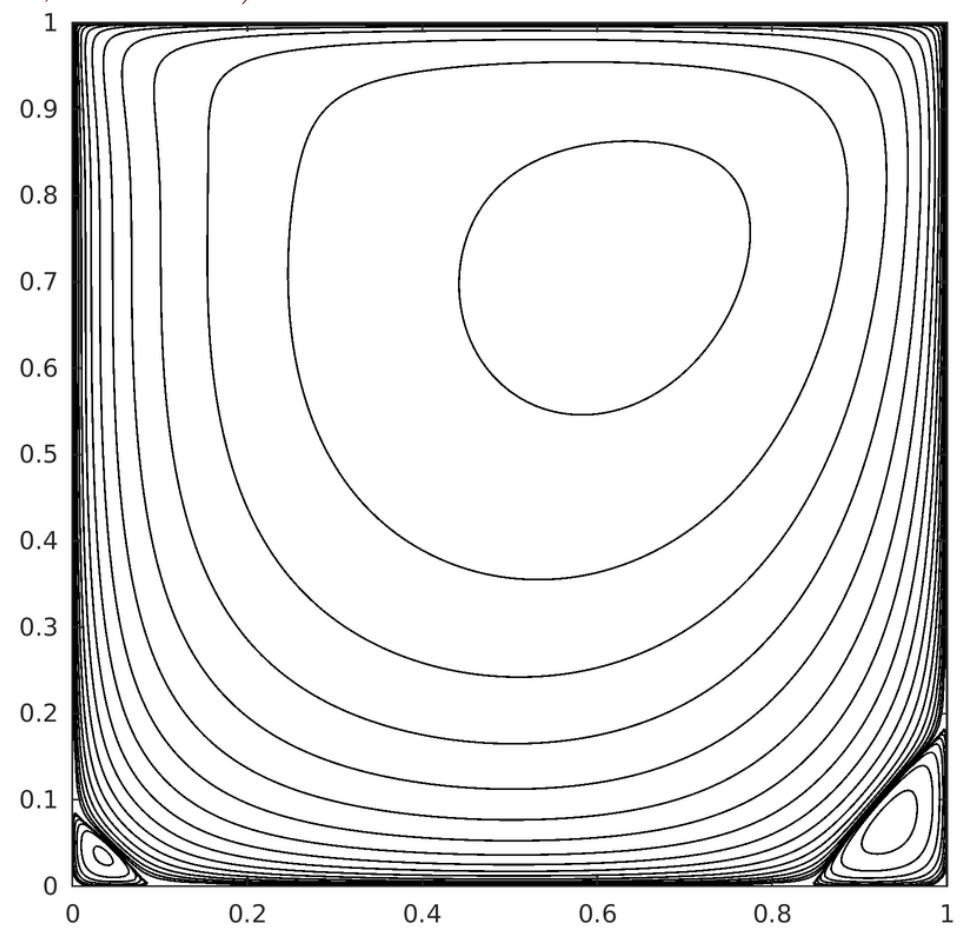


Table 5: Steady-state U(0.5, 0.5) spatial accuracy. uniform grids non-uniform grids

\begin{tabular}{rrrrr}
$\mathrm{N}$ & CDS-2 & Compact-4 & CDS-2 & Compact-4 \\
\hline 20 & -0.2309 & -0.2561 & -0.2524 & -0.2633 \\
40 & -0.2587 & -0.2670 & -0.2640 & -0.2671 \\
80 & -0.2653 & -0.2674 & -0.2666 & -0.2674 \\
160 & -0.2669 & -0.2675 & -0.2673 & -0.2675 \\
\hline $\mathcal{O}\left(\mathrm{h}^{\mathrm{n}}\right)$ & 2.0310 & 3.6247 & 2.0294 & 3.0448
\end{tabular}

is achieved. This means that the CDS-2 method can be used for the $\Pi_{x x}+\Pi_{y y}$ terms.

Figure 3 shows the lid-driven cavity streamlines obtained using the Compact- 4 scheme. This result is slightly different to that found by Brunei and Jouron [18] as we used the quadratically distributed driving velocity, equation (15). Table 5 shows grid tests results conducted using CDS- 2 and the Compact- 4 scheme with maximum residual of $1 \times 10^{-8}$ and both uniform and non-uniform grids, all obtained with CDS-2 for $\Pi_{x x}+\Pi_{y y}$. Results on the uniform grid show the CDS-2 scheme has very close to second-order accuracy, while the Compact- 4 scheme has slightly less than fourth-order accuracy. The Compact4 scheme provides a significantly more accurate solution, with the result obtained on the $40 \times 40$ grid approximately equal to that of the CDS- 2 scheme on the $160 \times 160$ grid. On the non-uniform grid the CDS-2 scheme is again very close to second-order, while Compact-4 is approximately third-order. Compact-4 is apparently more affected by the non-uniform error discussed in Section 2.3. Again the Compact-4 scheme achieves approximately the same accuracy on the $40 \times 40$ grid as the CDS- 2 scheme achieves on the $160 \times 160$ grid.

Figure 4 shows the convergence of the Navier-Stokes equation solutions with the error being the difference between the test solution and the $160 \times 160$ solution. This figure again shows the considerably improved accuracy and higher rates of convergence of the Compact- 4 scheme compared to the CDS-2 
Figure 4: Navier-Stokes equations solution convergence.

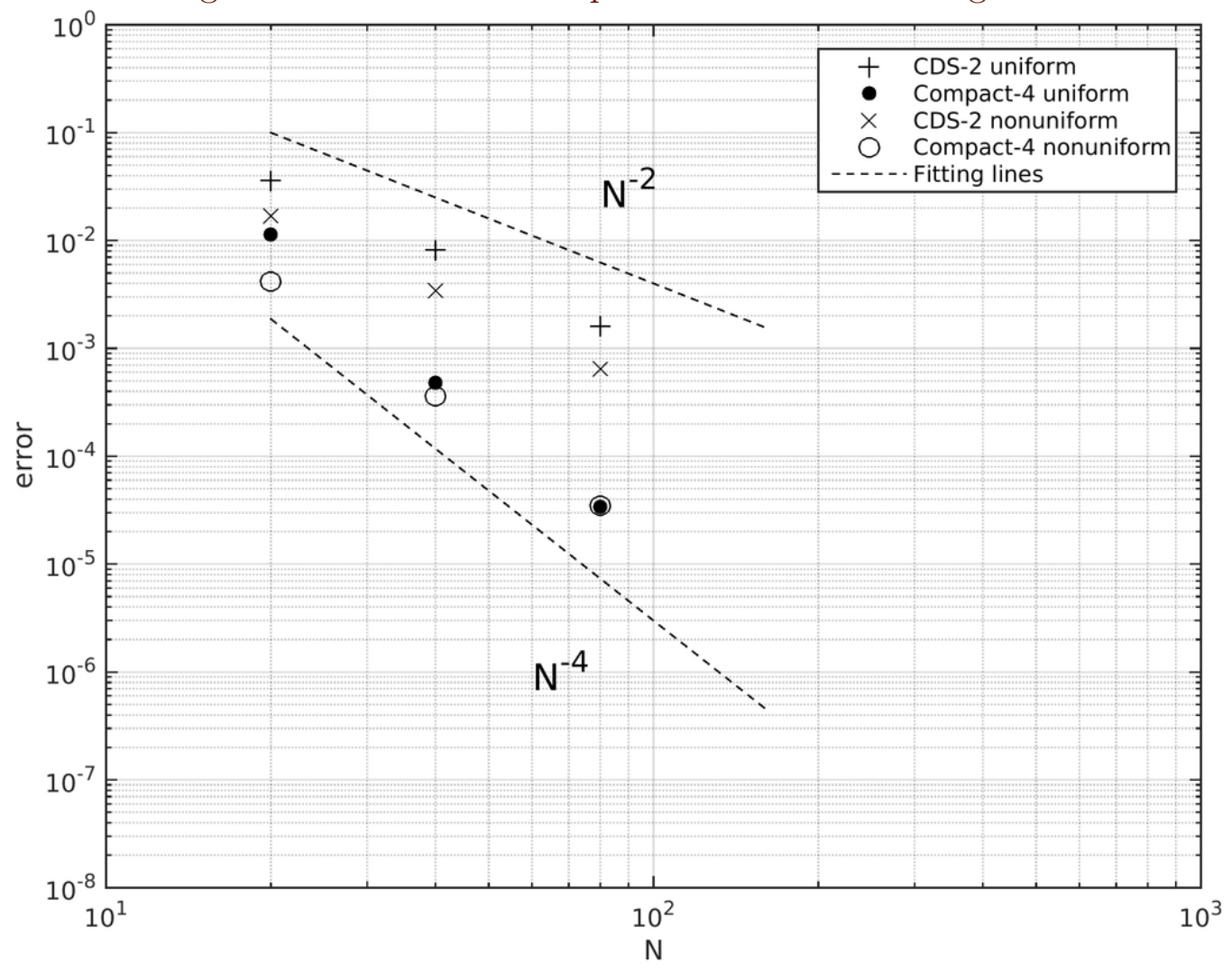

scheme, for both uniform and non-uniform grids. Examining all solution values we see that CDS- 2 is the least accurate scheme in all cases. CDS- 4 usually generates a slightly more accurate solution than the Compact- 4 scheme (except on the $20 \times 20$ grid). The better accuracy of CDS- 4 is because this scheme has a smaller coefficient for the sixth-order derivatives in the truncation series for first derivatives at boundary nodes (Table 1) compared to the Compact-4 scheme. However, this difference is only observed in the sixth decimal place on the $160 \times 160$ grid. Also, solutions on non-uniform grids are more accurate than their corresponding values on uniform grids, confirming that the use of 
refined grids in the boundary layer regions considerably improves solution accuracy.

\section{Summary}

Accurate solutions of the Navier-Stokes equations are required for direct or large-eddy simulation of turbulent or transition flows. Current high order schemes require large computational molecules or require the inversion of an additional matrix system, reducing the ease of coding and solution efficiency. The fourth-order spatial compact scheme proposed here uses fewer nodes without requiring an additional matrix system. The basic idea is to achieve high order by iteratively applying a low order scheme. It also allows a less complicated boundary treatment as well as an easier application on nonuniform grids. The new scheme was tested on the heat equation where the order of accuracy is validated, and was subsequently included in a full NavierStokes solver for the lid-driven cavity problem. The scheme was shown to be applicable and accurate for these two problems.

\section{References}

[1] A. J . Chorin. Numerical solution of the Navier-Stokes equations. Math. Comput., 22:745-762, 1968.doi:10.1090/S0025-5718-1968-0242392-2 C482

[2] J. H. Ferziger and M. Perić. Computational methods for fluid dynamics, pp. 42-46, 49. Springer, 1999. doi:10.1007/978-3-642-56026-2 C483, $\mathrm{C} 487$

[3] F. Gibou and R. Fedkiw. A fourth order accurate discretization for the Laplace and heat equations on arbitrary domains, with applications to the Stefan problem. J. Comput. Phys. 202:577-601, 2005. doi:10.1016/j.jcp.2004.07.018 C483 
[4] S. K. Lele. Compact finite difference schemes with spectral-like resolution. J. Comput. Phys. 103:16-42, 1992. doi:10.1016/0021-9991(92)90324-R C483, C485

[5] J. Zhao, W. Dai, and T. Niu. Fourth-order compact schemes of a heat conduction problem with Neumann boundary conditions. Numer. Meth. Part. D. E. 23:949-959, 2007. doi:10.1002/num.20200 C483

[6] S. Laizet and E. Lamballais. High-order compact schemes for incompressible flows: A simple and efficient method with quasi-spectral accuracy. J. Comput. Phys. 228(16):5989-6015, 2009. doi:10.1016/j.jcp.2009.05.010 C483

[7] P. C. Chu, C. Fan. A three-point combined compact difference scheme. J. Comput. Phys.140:370-399, 1998. doi:10.1006/jcph.1998.5899 C483, $\mathrm{C} 485$

[8] J. D. Hoffman. Numerical methods for engineers and scientists, pp. 271. CRC Press, 2001. https://www.crcpress.com/ Numerical-Methods-for-Engineers-and-Scientists-Second-Edition, Hoffman-Hoffman-Frankel/p/book/9780824704438 C484

[9] L. D. Kudryavtsev and M. K. Samarin. Lagrange interpolation formula. Encyclopedia of mathematics, 2016. http://www. encyclopediaofmath.org/index.php?title=Lagrange_ interpolation_formula\&oldid=17497. C485

[10] H. J. Crowder and C. Dalton. Errors in the use of non uniform mesh systems. J. Comput. Phys. 7:32-45, 1971. doi:10.1016/0021-9991(71)90047-7 C487

[11] E. Kálnay de Rivas. On the use of non-uniform grids in finite difference equations. J. Comput. Phys. 10:202-210, 1972. doi:10.1016/0021-9991(72)90060-5 C487

[12] A. E. P. Veldman and K. Rinzema. Playing with non-uniform grids. J. Eng. Math. 26:119-130, 1992. doi:10.1007/BF00043231 C487 
[13] P. E. O. Buelow, S. Venkateswaran, and C. L. Merkle. Effect of grid aspect ratio on convergence. AIAA J. 32:2401-2408, 1994. doi:10.2514/3.12306 C487

[14] J. Crank and P. Nicolson. A practical method for numerical evaluation of solutions of partial differential equations of the heat-conduction type. Proc. Camb. Phil. Soc. 43:50-67, 1947. doi:10.1007/BF02127704 C489

[15] J. G. Charney, R. Fjortoft, and J. von Neumann. Numerical integration of the barotropic vorticity equation. Tellus, 2:237-254, 1950. doi:10.1111/j.2153-3490.1950.tb00336.x C489

[16] O. Botella and R. Peyret. Benchmark spectral results on the lid-driven cavity flow. Comput. Fluid, 27:421-433, 1998. doi:10.1016/S0045-7930(98)00002-4 C492

[17] G. B. Deng, J. Piquet, P. Queutey, and M. Visonneau. Incompressible flow calculations with a consistent physical interpolation finite volume approach. Comput. Fluids, 23:1029-1047, 1994. doi:10.1016/0045-7930(94)90003-5 C492

[18] C.-H. Brunei and C. Jouron. An efficient scheme for solving steady incompressible Navier-Stokes equations, J. Comput. Phys. 89:389-413, 1990. doi:10.1016/0021-9991(90)90149-U C492, C496

[19] U. Ghia, K. N. Ghia, and C. T. Shin. High-Re solutions for incompressible flow using the Navier-Stokes equations and a multigrid method. J. Comput. Phys. 48:387-411, 1982. doi:10.1016/0021-9991(82)90058-4 C492

[20] R. Peyret and T. D. Taylor. Computational methods for fluid flow. Springer, 1983. doi:10.1007/978-3-642-85952-6 C492

[21] S. W. Armfield and R. Street. The fractional-step method for the Navier-Stokes equations on staggered grids: The accuracy of three variations. J. Comput. Phys. 153:660-665, 1999. doi:10.1006/jcph.1999.6275 C492 
[22] S. W. Armfield and R. Street. Fractional step methods for the Navier-Stokes equations on non-staggered grids, ANZIAM $J$. 42:134-156, 2000. http://journal.austms.org.au/ojs/index.php/ ANZIAMJ/article/view/593 C492

[23] S. W. Armfield and R. Street. An analysis and comparison of the time accuracy of fractional-step methods for the Navier-Stokes equations on staggered grids, Int. J. Numer. Meth. Fluids, 38:255-282, 2002. doi:10.1002/fld.217 C492

\section{Author addresses}

1. L. Zhou, School of Aerospace, Mechanical and Mechatronic Engineering, The University of Sydney, NSW 2006, Australia mailto:lzho3397@uni.sydney.edu.au

2. S. W. Armfield, School of Aerospace, Mechanical and Mechatronic Engineering, The University of Sydney, NSW 2006, Australia mailto:steven.armfield@sydney. edu.au 